\title{
EXTRAÇÃO SELETIVA DE $\mathrm{CO}_{2}$ DE DOLOMITAS E MAGNESITAS COEXISTENTES PARA ANÁLISE DOS ISÓTOPOS ESTÁVEIS DE CARBONO E OXIGÊNIO EM ROCHA
}

\author{
M.Z.Moreira' ${ }^{1}$ \& T.I.R.Almeida ${ }^{2}$
}

PALAVRAS-CHAVE: isótopos estáveis, ataque ácido, magnesita, dolomita.

MOREIRA, M.Z.; ALMEIDA, T.I.R. (1993) Extração seletiva de $\mathrm{CO}_{2}$ de dolomitas e magnesitas coexistentes para análise dos isótopos estáveis de carbono e oxigênio em rocha, Bol.IG-USP, Sér.Cient., 24:59-65.

\section{RESUMO}

A extração quimica seletiva de $\mathrm{CO}_{2}$ em amostras multicarbonatos é essencial para determinar corretamente as razóes isotópicas de carbono e oxigênio. Em trabalho recente. com numerosos dados experimentais sobre carbonatos puros, precipitados em laboratório, questiona-se os procedimentos laboratoriais tradicionais, sugerindo baixa eficiência na extraçăo de gás e alto risco de contaminaçăo entre as extraçōes das várias fases carbonáticas. O presente trabalho apresenta dados isotópicos obtidos segundo os procedimentos tradicionais em amostras de um depósito de magnesita proterozóico do tipo Veitsch, situado nas proximidades de Brumado, Bahia. Estes dados sâo compativeis com os disponiveis na literatura para depósitos semelhantes. Uma das amostras estudadas, de elevada razão $\mathrm{Mg} / \mathrm{Ca}$ (223) e virtualmente composta por magnesita, como confirmado por espectrometria de raio X, foi considerada como "padrão natural" de magnesita. Esta amostra foi submetida a experimentos destinados a testar a velocidade c eficiência de reação com $\mathrm{H}_{3} \mathrm{PO}_{4}$ a $100 \%$ a 25 e $50^{\circ}$ C. Um padrăo de calcário dolomitico natural foi também estudado segundo os procedimentos tradicionais, visando verificar o risco de contaminação de gás extraido de magnesita pela fraçăo dolomítica năo aberta. Os resultados obtidos mostram, por um lado, a eficiência do ataque de magnesita pelo método tradicional mas, por outro, confirmam o risco de contaminação entre fases minerais, principalmente $\mathrm{em}$ amostras ricas $\mathrm{cm}$ dolomita e pobres em magnesita.

\section{ABSTRACT}

The selective chemical extraction of $\mathrm{CO}_{2}$ is essential for correct measurements of isotopic compositions in rocks containig multiple carbonates. Recent experimental work with pure and artificial samples by Al-Aasm on acid attack for isotopic analysis suggests that traditional laboratory procedures are not efficient and that there is a real danger of contamination of the gases extracted from different coexisting carbonates. The present paper discusses data obtained by traditional methods on the $8^{13} \mathrm{C}$ and $8^{18} \mathrm{O}$ of a Proterozoic Veitsch-type magnesite deposit near Brumado, southern Bahia, Brazil. The new data are compatible with those

${ }_{1}^{1}$ Centro de Energia Nuclear na Agricultura/USP. Piracicaba, Brasil.

2 Departamento de Geologia Econōmica e Geofisica Aplicada, Instituto de Geociéncias/USP, Sảo Paulo, Brasai. 
reported in the literature for similar rocks. One of the studied samples exhibited a very high $\mathrm{Mg} / \mathrm{Ca}$ ratio (223) and was virtually all magnesite, as confirmed by X-ray spectrometry. This sample was considered a "natural standard"of magnesite for several laboratory experiments to test the speed of reaction of dolomite and magnesite with $\mathrm{H}_{3} \mathrm{PO}_{4}$ at 25 and $50^{\circ} \mathrm{C}$ in order to verify the best analytical procedures for obtaining carbon and oxygen isotopic ratios in magnesite ores. The problem of contamination is investigated in this same sample, with complementary studies with a dolostone sample. The results with the magnesite sample point to the application of traditional procedures but the dolostone data confirms the danger of contamination, principally in samples rich in dolomite and poor in magnesite.

\section{INTRODUÇÃO}

A obtenção de medidas precisas da composição isotópica de carbono e oxigênio de diferentes carbonatos coexistentes é essencial para a correta interpretação deste tipo de dados. $\mathrm{Na}$ maior parte dos casos, a separação fisica de diferentes carbonatos coexistentes é dificil ou improvável, justificando a tendência generalizada de se aplicar a extração química seletiva do $\mathrm{CO}_{2}$. Assim, desde McCrea (1950), muitos autores vem seguindo e desenvolvendo este método, como Friedman \& O'Neil (1977), que apresentaram uma revisão dos procedimentos laboratoriais para ataques ácidos dos carbonatos naturais mais comuns (calcita-dolomita-magnesitasiderita). Recentemente, dentro desta linha de pesquisa, Al-Aasm et al. (1990) apresentaram dados experimentais obtidos em carbonatos puros, precipitados em laboratório. A interpretaçāo feita por aqueles autores conduz a concluir pela necessidade de ataques ácidos notadamente mais vigorosos que os habitualmente aplicados, muito contrastantes, portanto, com os presentes na literatura, obtidos em carbonatos naturais. Os dados apresentados naquele trabalho, se por um lado sugerèm a adoção de procedimentos muitas vezes imcompativeis com a rotina laboratorial, por outro mostram o perigo de contaminação inter-carbonatos, devido à abertura parcial das várias fases carbonáticas presentes.

Este trabalho apresenta, como referência, razões isotópicas de carbono e oxigênio de 8 amostras de razão $\mathrm{Mg} / \mathrm{Ca}$ muito variada, compostas basicamente por dolomita e magnesita, com traços de calcita e siderita. As amostras foram coletadas no depósito de magnesita do tipo Veitsch de Campo de Dentro, na Serra das Éguas, Bahia, de idade proterozóica. Os procedimentos referidos por Friedman \& O'Neil (1977) foram adotados para a extração de gás de dolomita e magnesita destas amostras e os resultados são compativeis com os dados da literatura para este tipo de rocha. Uma destas amostras, altamente magnesiana (razão $\mathrm{Mg} / \mathrm{Ca}=223$ ) e composta virtualmente por magnesita, foi selecionada como padrão natural. Esta amostra foi submetida a ensaios para verificar a replicação de dados de Al-Aasm et al. (1990) bem como a eficiência dos procedimentos tradicionais e a possibilidade de contaminação em ataques ácidos à temperatura ambiente. Um padrão de calcário dolomítico foi também estudado pelos procedimentos tradicionais, confirmando os riscos de contaminação por abertura parcial de carbonatos, como sugeridos por aqueles autores.

\section{MATERIAIS E MÉTODOS}

Almeida (1990) originalmente coletou e estudou oito amostras de magnesititos em testemunhos de sondagem rotativa executados no Depósito de Campo de Dentro. Este depósito de idade proterozóica 
foi metamorfisado no fácies anfibolito alto, como indicam as paragêneses das encaixantes. Foram selecionadas amostras com razões $\mathrm{Mg} / \mathrm{Ca}$ variando de 23,4 a 223, moidas a $150 \#$ e parcialmente analisadas por difratometria de raio $\mathrm{X}$, para verificação de sua mineralogia. As análises isotópicas foram feitas em espectrômetro de massas Mat Delta E Finnegan com precisão de 0,1\%o contra padrảo PDB (Belemnitella americana, Peedee Formation). Os resultados em $\delta \%$ estão expressos para oxigênio em SMOW para melhor comparação com dados da literatura e a transformação foi feita segundo a equação de Friedman \& O'Neil (1977)

$$
{ }^{18} \mathrm{O}_{\text {SMOW }}=\delta^{18} \mathrm{O}_{\text {PBD }} \times 1,03086+30,86
$$

e os resultados estão apresentados como $\delta$ por mil para carbono e SMOW para oxigênio. Os fatores utilizados para correção do fracionamento nas reaçôes ácido-carbonato foram as seguintes: 1,0125 (calcita, 25 ${ }^{\circ} \mathrm{C}$ ); 1,01108 (dolomita, $25^{\circ} \mathrm{C}$ ) e 1,01160 (magnesita, $50^{\circ} \mathrm{C}$ ), sempre de acordo com Friedman \& O'Neil (1977). Para correção do fracionamento cinético dependente da temperatura, foram subtraidos $0,83 \%$ e $1,28 \%$ dos $\delta^{18} \mathrm{O}$ respectivamente das razōes referentes a dolomitas e magnesitas (Aharon, 1988).

Para a extração de $\mathrm{CO}_{2}$ de calcita e dolomita, foi pesada uma aliquota de 50 $\mathrm{mg}$, atacada, em vácuo, por $5 \mathrm{ml} \mathrm{de} \mathrm{H}_{3} \mathrm{PO}_{4}$ a $100 \%$ e a uma temperatura de $25^{\circ} \mathrm{C}$, com extração de gás após uma hora (representando, em princípio, gás de calcita), continuando a reação por 72 horas, sendo então extraido o gás considerado como referente à dolomita (McCrea, 1950). Para atacar a magnesita, foi utilizado o procedimento proposto por Perry \& Tan (1972), com a reação persistindo por 72 horas adicionais, a uma temperatura de $50^{\circ} \mathrm{C}$

Os ensaios feitos sobre a amostra de elevada razão $\mathrm{Mg} / \mathrm{Ca}$, assumida como padrão natural de magnesita após a extração de dolomita, se iniciaram com exame por difratometria de raio $\mathrm{X}$, que confirmou a análise quimica, indicando apenas a presença de magnesita. A amostra foi atacada por $\mathrm{H}_{3} \mathrm{PO}_{4}$ a $50^{\circ} \mathrm{C}$, com extrações após 24,96 e 196 horas. Para examinar a presença e intensidade de reação a $25^{\circ} \mathrm{C}$, cinco outras aliquotas desta amostra foram atacadas a esta temperatura, com extrações sendo feitas a, respectivamente, $4-12-22,5$ horas; $24-360$ e 769 horas; 48 e 1144 horas; 72 horas e 144 horas. Finalmente, para testar a contaminação entre as fases carbonáticas, uma amostra padrão de calcário dolomítico (IPT 248) foi moida a $150 \#$ e analisada pelo procedimento de McCrea (1950) anteriormente descrito.

\section{RESULTADOS OBTIDOS E DISCUS- SÃO}

As razões isotópicas de carbono e oxigênio das amostras de magnesititos do Depósito de Campo de Dentro estão apresentadas como referência na Tabela 1 , bem como as dosagens em $\mathrm{Ca}$ e $\mathrm{Mg}$, obtidas por ICP-AES no CENA. Estes resultados isotópicos mostraram-se compativeis com os dados disponiveis na literatura e obtidos para rochas similares a partir dos mesmos procedimentos laboratoriais, como exposto na Figura 1.

O diagrama da Figura 1 mostra que os resultados produzidos pelos laboratórios do CENA replicam os presentes na literatura para depósitos semelhantes. Por outro lado, os resultados obtidos com o padrão natural de magnesita, não replicam os dados de Al-Aasm et al. (1990), como apresentado na Tabela 2 . Em ataque a $50^{\circ} \mathrm{C}$, foi obtido gás já a 24 horas, sem atingir a estabilidade isotópica para o carbono, indicando eventual contaminação, embora a estabilidade do oxigênio tenha sido atingida 
Tabela 1 - Composição em $\mathrm{Mg}$ e Ca em \%, razões $\mathrm{Mg} / \mathrm{Ca}$ e $\delta^{13} \mathrm{C}_{(\mathrm{PDB})}$ and $\delta^{18} \mathrm{O}_{(\mathrm{SMOW})}$ em permil das amostras de magnesititos do Depósito de Campo de Dentro.

\begin{tabular}{|c|c|c|c|c|c|c|}
\hline $\mathrm{Ca}(\%)$ & $\mathrm{Mg}(\%)$ & $\mathrm{Mg} / \mathrm{Ca}$ & $\begin{array}{l}\text { dolomita } \\
8^{13} \mathrm{C}_{\text {(pDs }}\end{array}$ & $\begin{array}{l}\text { dolomita } \\
8^{18} \mathrm{O}_{\text {(smow) }}\end{array}$ & $\begin{array}{r}\text { magnesita } \\
\left.8^{13} \mathrm{C}_{(\mathrm{D} B}\right)\end{array}$ & $\begin{array}{l}\text { magnesita } \\
8^{18} \mathrm{O}_{\text {(smom) }}\end{array}$ \\
\hline 1,73 & 23,4 & 14 & $-0,71$ & 11,61 & $-0,36$ & 10,05 \\
\hline 1,42 & 22,7 & 16 & $-0,66$ & 12,23 & $-0,30$ & 10,85 \\
\hline 0,73 & 22,9 & $3 \mathrm{I}$ & $-0,57$ & 12,08 & $-0,29$ & 10,65 \\
\hline 0,46 & 24,4 & 53 & $-0,86$ & 12,42 & $-0,62$ & 11,09 \\
\hline 0,23 & 26,0 & 113 & $-0,90$ & 12,53 & $-0,23$ & 10,40 \\
\hline 0,21 & 26,2 & 125 & $-0,87$ & 12,66 & $-0,54$ & 10,33 \\
\hline 0,19 & 24.3 & 128 & $-1,41$ & 11,56 & $-0,54$ & 10,32 \\
\hline 0,12 & 26,7 & 223 & $-0,48$ & 12,86 & $-0,32$ & 11,00 \\
\hline
\end{tabular}

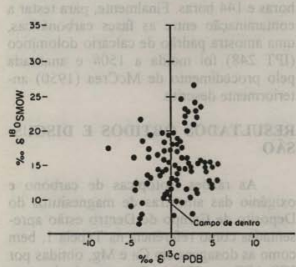

Figura 1 - Diagrama $8^{13} \mathrm{C}_{\mathrm{PDB}} \times 8^{18} \mathrm{O}_{\mathrm{SMOW}}$ de magnesitas de magnesititos, Dados disponiveis na literatura e a composiçâo média do Depósito de Campo de Dentro: $\delta^{13} \mathrm{C}=-0,38$ e $\delta^{18} \mathrm{O}=10,52$ (Modificado de Kralik et al., 1989).

já a esse tempo. Na extração feita após 96 horas, verifica-se estabilidade isotópica de ambos os elementos, sem evidências de contaminação, o que implica em abertura inequívoca de magnesita, o que pode ser comprovado pelas razões isotópicas da extracão feita a 196 horas, enquanto os dados de Al-Aasm et al. (1990) indicam um mínimo de 240 horas a essa temperatura para análises isotópicas acuradas deste mineral. A maior vulnerabilidade da amostra natural ao ataque ácido, se comparada à
Tabela 2 - Produção de $\mathrm{CO}_{2}$ ( $\mu$ moles) e composição isotópica de carbono (PDB) e oxigênio (SMOW) a partir de amostra natural de magnesita moída abaixo de $150 \#$. Ataque por $\mathrm{H}_{3} \mathrm{PO}_{4}$ a $100 \%$ e a $50^{\circ}$ C.

TEMPO PRODUCCÃO $\mathrm{CO}_{2} \quad \delta^{13} \mathrm{C} \quad \delta^{18} \mathrm{O}$ (horas) ( $\mu$ moles $\mathrm{CO}_{2}$ )

$\begin{array}{cccc}24 & 59 & -0,63 & 10,85 \\ 96 & 164 & -0,35 & 10,31 \\ 169 & 230 & -0,30 & 10,32\end{array}$

das amostras puras, estudadas por $\mathrm{Al}$-Aasm et al. (1990), deve-se, possivelmente, à inevitável presença de microcomponentes em carbonatos naturais (Kolthoff et al., 1989), introduzindo distorçōes nas celas unitárias e as tornando menos resistentes a ataques ácidos.

Em estudo semelhante, para obter as composições isotópicas de dolomitas e magnesitas, Aharon (1988) verificou que os procedimentos tradicionais mostravam-se adequados, observando variaçōes de $0,15 \%$ nas razões isotópicas de carbono e oxigênio em extrações feitas a 48, 72 e 96 horas, com abertura de apenas 19 a $73 \%$ da amostra. Os dados ora apresentados replicam os daquele autor, uma vez que verificaram-se pequenas diferenças $(0,35 \%)$ ou variações abaixo do erro analítico, entre extraçø̃es feitas entre 24 e 169 horas. 
Verificou-se, entretanto, uma liberação de gás mais lenta que a encontrada por Aharon (1988), com apenas $42 \%$ da amostra sendo aberta após 169 horas de ataque ácido. Pela curva da Figura 2, apenas $26 \%$ da amostra teria sido atacada após 72 horas, tempo para extração adotado internacionalmente. Observe-se que Al-Aasm et al. (1990) mostram a abertura de cerca de $12 \%$ apos 72 horas para amostras moidas a $<200 \#$ e apenas $6 \%$ para granulometria entre $100 \mathrm{e} 140 \#$.

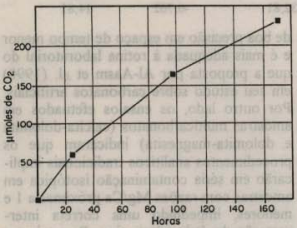

Figura 2 - Produçăo parcial de $\mathrm{CO}_{2}$ na reaçăo de magnesita com $\mathrm{H}_{3} \mathrm{PO}_{4}$ em funçẳo do tempo. Temperatura de reaça: $50^{\circ} \mathrm{C}$. Granulometria de moagem a $150 \#$

Os ensaios desenvolvidos sobre a amostra considerada como padrão natural de magnesita, em reação a $25^{\circ} \mathrm{C}$ em cinco sub-amostras iguais, geraram os dados expostos na Tabela 3.

Os resultados mostram um ataque muito lento da magnesita, com evidências de contaminação, na primeira extração, por traços de calcita e/ou dolomita de inevitável presença em carbonatos de origem natural. A estabilidade isotópica foi relativamente atingida após 144 horas e, mesmo após 1174 horas de reação a $25^{\circ} \mathrm{C}$, apenas $15 \%$ da amostra foi aberta. Os dados demonstram assim a pequena importância da contaminação por extração involuntária de
$\mathrm{CO}_{2}$ de magnesita, durante a extração de gás de dolomita e calcita, pelo procedimento de McCrea (1950).

A aparente estabilidade na produção de gás a $25^{\circ} \mathrm{C}$ pode dever-se ao aumento da pressão no tubo de reação, sugerindo a necessidade de remoção simultânea de $\mathrm{CO}_{2}$, para completar a reação e atingir a estabilidade isotópica (Fig. 3). A necessidade de temperaturas mais elevadas para um ataque ácido eficiente de magnesita fica evidente, corroborando procedimentos como os adotados por Cornides \& Kusakabe (1977), que fizeram a reação a $95^{\circ} \mathrm{C}$.

O padrão de calcário dolomítico IPT 248 sofreu ataque acido segundo os procedimentos tradicionais de McCrea, verificando-se, após 72 horas de reação, abertura de $81 \%$ da amostra. Embora o residuo sólido nāo tenha sido estudado, é evidente que mais de $20 \%$ da dolomita presente não foi aberta em 72 horas a $25^{\circ} \mathrm{C}$, tendo em vista que a calcita presente foi prioritariamente digerida no ataque. Isto implica em obter-se razões isotópicas de carbonatos menos solúveis (siderita, magnesita) com erros proporcionais ao teor de dolomita que é apenas parcialmente extraida. Nos dados de Almeida (1990) esta contaminação certamente ocorreu, porém não de forma a prejudicar seriamente o conjunto de dados, uma vez que a dolomita ocorria em baixas porcentagens na maior parte das amostras. Verifica-se na Tabela 1, entretanto, que a diferença entre as composiçōes isotópicas de carbono e oxigênio de dolomitas e magnesitas é menor nas amostras mais dolomiticas do que naquelas de elevada razão $\mathrm{Mg} / \mathrm{Ca}$, o que pode dever-se à contaminaçăo. Recomenda-se, portanto. para amostras de multicarbonatos calcitadolomita-magnesita, um ataque ácido adicional (desprezando-se o gás extraido), de 2 horas a $25^{\circ} \mathrm{C}$ para eliminar residuos de calcita e 2 horas a $50^{\circ} \mathrm{C}$ para dolomita. 
Tabela 3 - Tempo de reação (horas) com $\mathrm{H}_{3} \mathrm{PO}_{4}$ a $100 \%$ a $25^{\circ} \mathrm{C}$, produção de $\mathrm{CO}_{2}$ ( $\mu$ moles) e $8^{13} \mathrm{C}_{\mathrm{PDB}}$ e $8^{18} \mathrm{O}_{\mathrm{SMOW}}$. (* = dados inexistentes). Amostras moidas a $150 \#$.

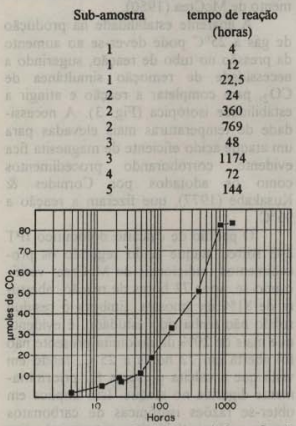

Figura 3 - Produção de $\mathrm{CO}_{2}$ (umoles) em função do tempo. Dados da Tabela 3 .

Outra importante variável é a granulometria das amostras após a moagem, aspecto também considerado por Al-Aasm et al. (1990) e por Almeida \& Wunder (1993). O primeiro trabalho indica uma granulometria mais fina (400\#) para possibilitar a abertura de magnesita, enquanto o último sugere um controle da granulometria entre 150 e $300 \#$, a qual permite ataque efetivo da magnesita e não apresenta abertura precoce de magnesita durante a extração de gás referente a dolomita.

\section{CONCLUSŌES}

A metodologia de ataque ácido tradicional produziu resultados considerados

$\begin{array}{ccc}\begin{array}{c}\text { produça de } \mathrm{CO}_{2} \\ \text { ( } \mu \text { moles) }\end{array} & \delta^{13} \mathrm{C} & 8^{18} \mathrm{O} \\ 1,79 & -3,71 & 21,17 \\ 5,35 & - & 18,08 \\ 9,09 & -1,60 & 16,36 \\ 7,42 & -1,66 & 16,22 \\ 50,58 & -0,612 & 14,36 \\ 82,35 & -0,306 & * \\ 11,49 & -1,92 & 16,50 \\ 83,17 & * & \\ 18,73 & -1,37 & 15,31 \\ 32,81 & -0,762 & 14,81\end{array}$

de boa precisão em espaço de tempo menor e é mais adequada à rotina laboratorial do que a proposta por Al-Aasm et al. (1990) em seu estudo sobre carbonatos artificiais. Por outro lado, os ensaios efetuados em amostras multicarbonatos (calcita-dolomita e dolomita-magnesita) indicaram que os procedimentos analiticos tradicionais implicarão em séria contaminação isotópica em amostras com razōes $\mathrm{Mg} / \mathrm{Ca}$ próximas a $1 \mathrm{e}$ menores, impedindo uma correta interpretação de dados deste tipo. Para tais casos indica-se uma extração extra e descarte de gás para climinar residuos de carbonatos mais solúveis não totalmente extraidos. Em amostras essencialmente magnesíticas, embora a possibilidade de contaminação sensivel seja pequena e insuficiente para mascarar as razões isotópicas, também se recomenda uma extração extra a $50^{\circ} \mathrm{C}$ e descarte do gás produzido, para maior confiabilidade do dado obtido.

\section{AGRADECIMENTOS}

Os autores agradecem as facilidades fornecidas para a execução deste trabalho pela IBAR NORDESTE S.A. (Grupo Votorantim) por intermédio de seu diretor, Dr. Clóvis Scripilitti, bem como ao Dr. Eichii Matsui pelas sugestōes e à Sra. Antônia Perez, pelos trabalhos laboratoriais. Este 
trabalho é uma contribuição ao International Correlation Programme 342 - Geochronology and Isotopic Characterization of Metallogenetic Provinces in South America.

\section{REFERÊNCIAS BIBLIOGRÁFICAS}

AHARON, P. (1988) A stable isotope study of magnesites from the Rum Jungle uranium field, Australia: implications for the origin of strata-bound massive magnesites. Chemical Geology, 69: 127-145.

AL-AASM, I.S.; TAYLOR, B.E.; SOUTH, B. (1990) Stable isotopes analysis of multiple carbonate samples using selective acid extraction. Chemical Geology, 80:119-125.

ALMEIDA, T.I.R. (1990) Magnesita do depósito de Campo de Dentro, Serra das Éguas, Bahia Geoquímica e Gênese. São Paulo, 153p. (Tese de Doutorado - Instituto de Geociências/ USP).

ALMEIDA, T.I.R.; WUNDER, E. (1993) Aplicação dos isótopos estáveis de carbono e oxigênio em carbonatos hipermagnesianos de Iguatu, Ceará: indicaçōes genéticas, discussões sobre a resistência diferencial de carbonatos frente a eventos metamórficos e ensaio metodológico. (Relatório de atividades de Iniciação Cientifica CNPQ/RUSP -
107.163/92-6. IGc/USP).

CORNIDES, L.; KUSAKABE, M. (1977) Preparation of carbon dioxide from magnesite for isotopic analysis. Frezentus Analytical Chemistry, 287:310311.

FRIEDMAN, I; O'NEIL, J.R. (1977) Compilation os stable isotope fractionation factors of geochemical interest. $\mathbf{U}$. S. Geological Survey Professional Paper, 440KK: $1-12$.

KOLTHOFF, I.M., SANDELL, E.B., MEEHAN, E.J; BRUCKENTEIN, S. (1989) Quantitative Chemical Analysis. 4. ed. London, MacMillan. 1199p.

KRALIK, M, AHARON, P,; SCHROLL, E; ZACHMANN, D. (1989) Carbon and oxygen isotope systematics of magnesites: a review. Monograph Series on Mineral Deposits, 28:232-254.

MCCREA, J.M. (1950) On the isotope geochemistry of carbonates and a paleotemperature scale. Journal of Chemical Physics, 74: 174-196.

PERRY, E.C.; TAN, F.C. (1972) Significance of oxygen and carbon isotope variation in Early Precambrian cherts and carbonate rocks of Southern Africa. Bulletin of the Geological Society of América, 83(3):647-664.

M.Z.Moreira - Centro de Energia Nuclear na Agricultura/USP - Piracicaba - Brasil

T.I.R.Almeida - Departamento de Geologia Econòmica e Geofisica Aplicada, Instituto de Geociências/USP -

Caixa Postal 11.348 CEP 05422-970 São Paulo, Brasil. 